\title{
Feature of Electron Tomography with Ultra-HVEM of Osaka University
}

\author{
Akio Takaoka Kyoko Matoba Toshiaki Hasegawa and Hirotaro Mori \\ Research Center for Ultra-high Voltage Electron Microscopy, \\ Osaka University, Suita, Osaka 565-0871, Japan; e-mail takaoka@uhvem.osaka-u.ac.jp
}

Although every object is composed of atoms or molecules, there exists a suitable size and resolution to analyze the structure; cell, organelle, compound macromolecule and molecule for a biological sample. In the case of functional materials such as LSI, it also subdivided into blocks, segments and crystalline. For 3D microscopy in various scales, X-ray or visible light is advantageous when the resolution is enough to a micrometer because of the high penetration in sample. To obtain a high resolution of nanometers, electron microscopes are suitable, but the sample thickness is restricted less than several hundreds nm in usual. The ultra-HVEM with 3MV in Osaka University is excellent in both the penetration and the resolution. We can observe $5 \mu \mathrm{m}$ slice tilted 60 degree and then the resolution holds in a value about 1/1000 of the thickness when the biological slice is well stained [1]. Fig. 1 shows the applicable dimension of sample and resolution for various CT methods. It is seen that ultra-HVEM CT is the most suitable method for 3D analysis of cell structure.

\section{Key factors in ultra-HVEM CT}

(1) In the preparation of sample, electron staining is quite important to get a fine 3D image because the contrast of micrographs becomes weak as the electron energy increases. Furthermore, the density of staining tends to change in depth, so it often differs between the surface and central area. We tested various kinds of solution and condition and found that 3\% uranyl acetate in $70 \%$ methanol solution is best under heating by a microwave oven in 30 seconds [2].

(2) Realization of little missing zone is important at the taking of a tilt series, so we developed a 360-degree rotating holder [3] and also we use a high quality $4 \mathrm{k}$ x 4k CCD camera. The shape of stained sample fairly resists against beam radiation except the shrinkage of whole size by several \% during the micrograph of a tilt-series.

(3) The performance of alignment between tilt-series strongly affects the resolution of reconstructed image; we need the geometrical modification to the shrinkage and the image rotation due to the deviation from eucentric condition. We use some gold particles as the marker and the excellent software IMOD developed in Colorado University.

\section{Application of ultra-HVEM tomography}

In the center, we collaborate with many researchers in various fields to observe the $3 \mathrm{D}$ structure; 1. Structure of unicellular organism such as bacteria and plankton (an example is shown in Fig.3).

2. Cell organelle such as nucleus, Golgi system, mitochondria, chromosome, etc.

3. Pathological injury texture such as nervous system, capillary, etc (shown in Fig.2).

4. Animal or plant section such as human hair, papilla cell, osteoblast, etc (shown in Fig.4).

5. Hybrid material between organic and inorganic matters.

6. Polymer such as block copolymer.

7. Electronic device such as LSI (shown in Fig5).

A part of this work was supported by "Nanotechnology Support Project" of the Ministry of Education, Culture, Sports, Science and Technology (MEXT), Japan. 
[1] A. Takaoka, S. Furukawa, Y. Murase and H. Mori; Proc. $15^{\text {th }}$ ICEM, Darban 2002, vol.3, p.389. [2] A. Takaoka, A. Kuwae and T. Hasegawa; Proc. $8^{\text {th }}$ Asia-Pacific Conf. on EM, Kanazawa 2004, p.168.

[3] H. Zhang, A. Takaoka and K. Miyauchi; Rev. Sci. Instrum, 69, 11, 4004, 1998
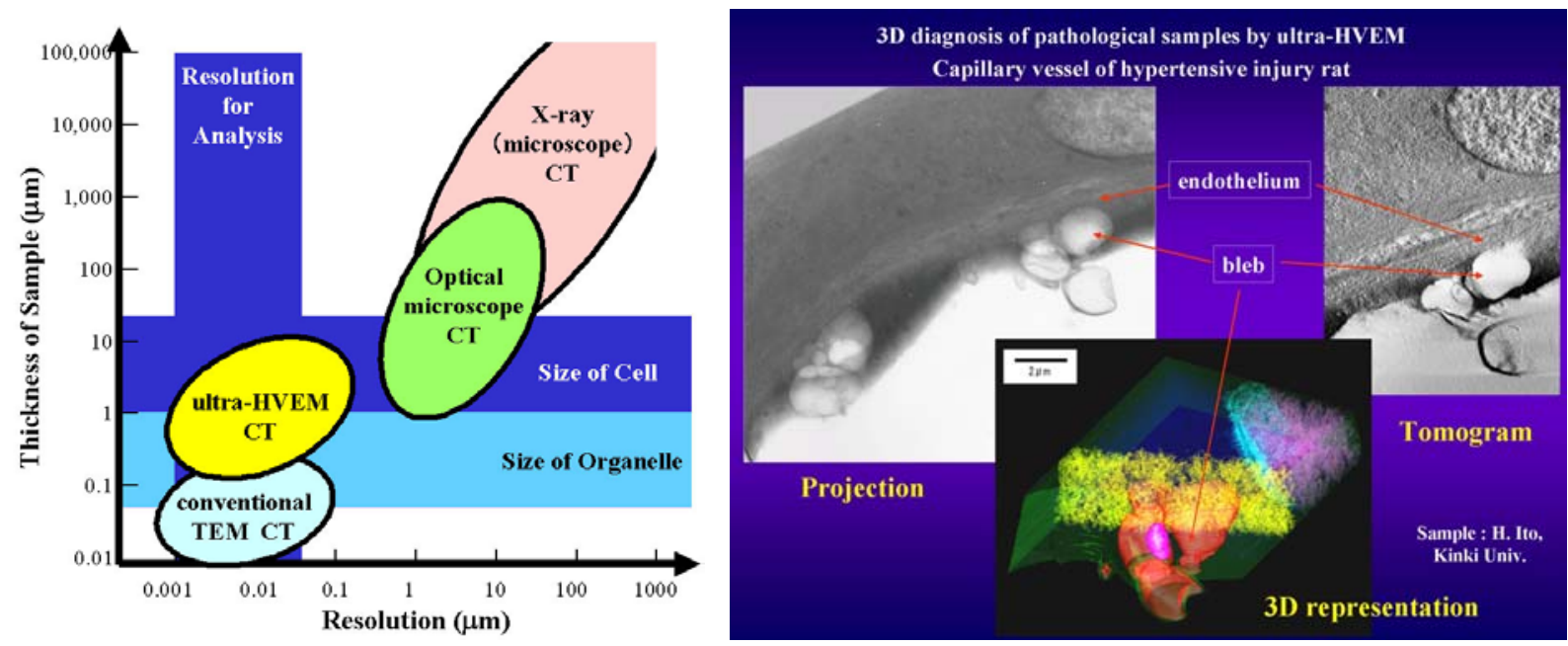

Fig.1 Applicable dimension for ultra-HVEM CT

Fig. 2 3D reconstruction of a capillary vessel of hypertensive rat. All blebs grow from the inner wall (endothelium), and a new bleb (pink) grows inside of an existing bleb (painted by red).

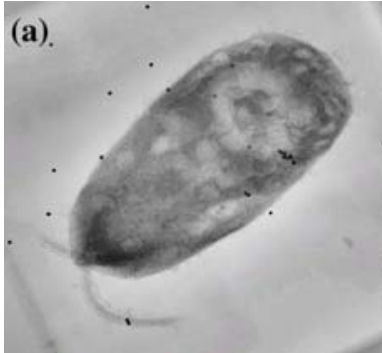

(b)

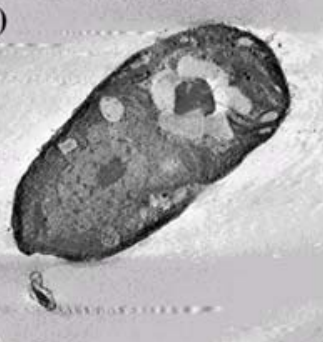

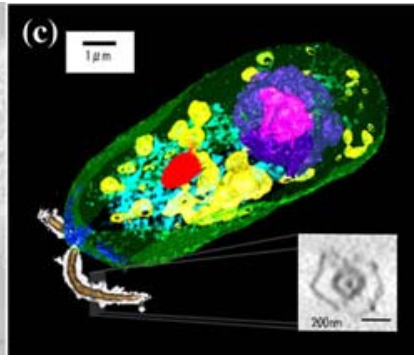

Fig.3 Unicellular plankton (Oocystis Apiculasta); (a) projection,(b) tomogram,(c) 3D reconstruction
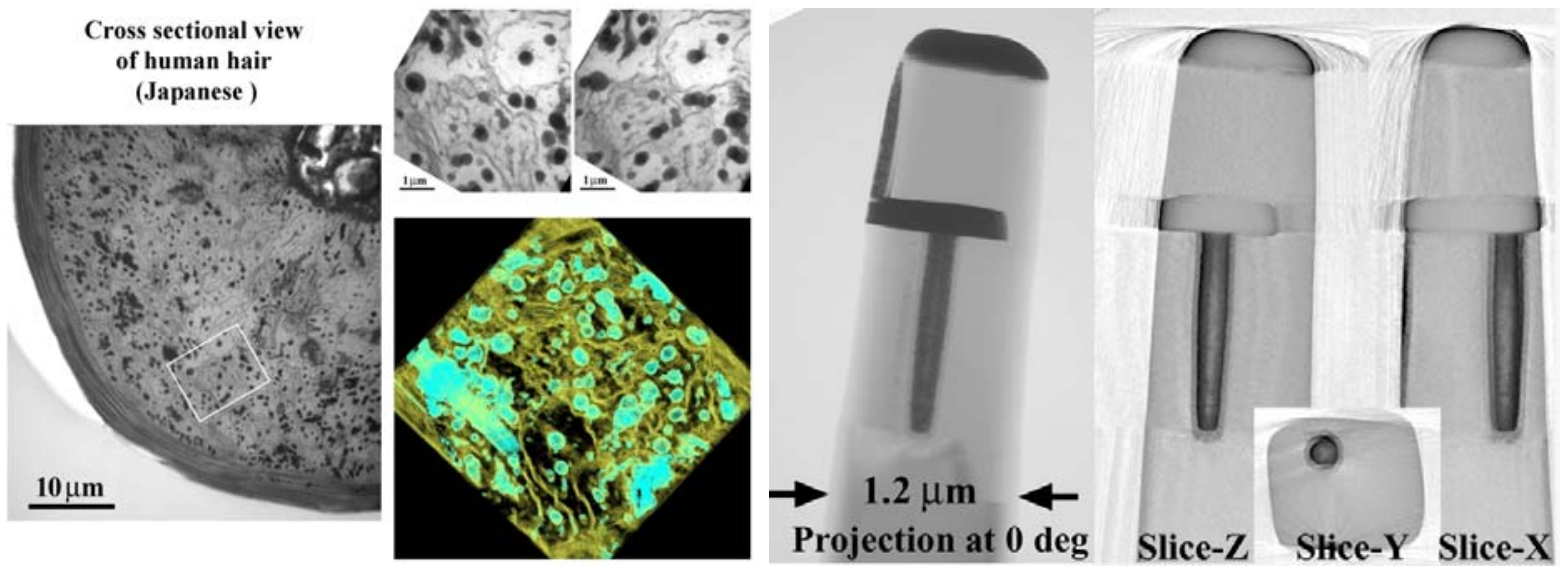

Fig.4 3D reconstruction of human hair fiber. Fig.5 Geometrical 3D-measurement of LSI structure. 